\title{
Human Histocompatibility Antigen Associations in Subacute Cutaneous Lupus Erythematosus
}

\author{
Richard D. Sontheimer, Peter Stastny, and James N. Gilliam, Department \\ of Internal Medicine, University of Texas Health Science Center at Dallas \\ Southwestern Medical School Dallas, Texas 75235
}

\begin{abstract}
A в S T R A C T We have identified a clinically distinct subset of lupus erythematosus patients marked by the presence of a histologically proven, nonscarring variety of cutaneous LE (subacute cutaneous LE) in which there is a very high frequency of the human leukocyte antigens (HLA) B8 and DR3. Differences in the configuration of their skin lesions allowed a separation of the patients into two clinical subgroups; annular and papulosquamous. HLA-B8 was increased in the annular subgroup $(81 \%$, corrected $P(P c)<0.007)$ and combined group $\left(65 \%, P_{c}<0.004\right)$. HLA-DR3 was present in all 11 of the annular patients $(100 \%, P c$ $<0.00008$ ). In addition, HLA-DR3 was present in increased frequencies in the papulosquamous subgroup $\left(60 \%, P_{c}<0.04\right)$ and combined group $(77 \%$, $\left.P_{\mathrm{c}}<0.00008\right)$. Thus, HLA-DR3 positive individuals have a relative risk of 10.8 for developing subacute cutaneous LE of either type and an even greater relative risk (67.1) for the annular variety. The HLA phenotype A1, B8, DR3 was also found more commonly in the annular $(73 \%, P<0.00008)$ and combined patient groups $(46 \%, P<0.004)$. These HLA associations, which are stronger than ever before reported for any form of LE, did not result from the concurrent presence of subclinical Sjögren's syndrome. Thus, subacute cutaneous LE can now be added to the growing list of HLA-B8, DR3-associated diseases that have autoimmune features.
\end{abstract}

\section{INTRODUCTION}

Lupus erythematosus $(\mathrm{LE})^{1}$ is a multisystem disease with many patterns of clinical expression. Autoanti-

Dr. Sontheimer is the recipient of a Clinical Investigator Award from the National Institutes of Health (AM-00730). Dr. Gilliam is the recipient of a Research Career Development Award from the National Institutes of Health AM-00278.

Received for publication 2.5 August 1980 and in revised form October 101.980 .

${ }^{1}$ Abbreviations used in this paper: LE, lupus erethematosus; $P$ c, corrected $P$ value; RR, relative risk; SCLE subacute cutaneous LE; SS, Sjögren's syndrome; SLE, systemic LE. body production regularly accompanies the expression of this disease process and it is suspected that most of the clinical manifestations of LE are in fact autoimmune mediated. Both twin and family studies $(1,2)$ have established that genetic factors exist in LE and an abnormality in immune regulation in relatives of $\mathrm{LE}$ patients was recently reported (3). Because of the evidence in animals that suggests that immune responsiveness (4) and some forms of autoimmunity (5) are under genetic control of the main histocompatibility complex it has been speculated that LE results from an interaction of genetic, immunologic, and environmental factors (6). However, data to support the hypothesis that predisposition for development of LE is based on main histocompatibility complex determined disorder of immune regulation has been slow to come forward. A partial explanation for this fact is that human leukocyte antigen (HLA) typing studies are difficult to carry out in this disease because of the frequent presence of lymphocytotoxic antibodies. In addition, the clinical heterogeneity of LE patients tends to blurr the results of HLA typing as there may also exist genetic heterogeneity. Despite these difficulties several groups of investigators have recently reported weak but significant HLA associations in systemic LE $(7-15)$.

We have recently studied a group of patients who had a clinically distinct, non-scarring variety of cutaneous LE that we have designated as subacute cutaneous LE (SCLE) (16). This form of cutaneous LE occurs in a characteristic distribution and is clinically and histologically distinct from discoid LE. Patients with SCLE often had similar patterns of mild systemic involvement and appeared to represent a distinct subset of LE, thus offering the possibility of performing HLA typing in a relatively homogeneous group of patients. We noted also that the majority of patients with SCLE were Caucasoid, in spite of the fact that a large proportion of the LE population at our institution is black, suggesting the possibility of an important genetic factor. The results to be presented 
indicate that an association with certain genes of the HLA system exists in this subset of LE patients which is stronger than any previously observed association in patients with LE.

\section{METHODS}

Patient selection. 26 patients with histologically confirmed SCLE as previously defined (16), who were available for HLA typing, were included in the present study. 11 patients were common to both the present study group and the original group reported (16). Based on the configuration of their skin lesions these patients could be divided into annular and papulosquamous subgroups (16). There were 11 patients in the annular group and 15 in the papulosquamous group. All patients were examined by Dr. Sontheimer and Dr. Gilliam and were categorized as either annular or papulosquamous before laboratory testing or HLA typing studies were carried out.

HLA typing. HLA-A, -B and -DR type were determined serologically by methods that have been previously described (17). The patients were tested for 19 HLA-A, 31 HLA-B, and 8 HLA-DR antigens. Our normal controls were all Caucasoids, living in the same geographic area as the SCLE patients and typed in our laboratory using the same reagents. The HLA data were analyzed for significance by the chisquare test with the Yates' correction. The $P$ values obtained were corrected by multiplying by the number of antigens determined at the locus $(P c)$. The relative risks (RR) were calculated by either the formula of Woolf or that of Haldane as appropriate.

Evaluation for Sjögren's syndrome. 17 of the SCLE patients completed a questionnaire in which five questions were asked regarding the presence or absence of dry eyes or dry mouth. An affirmative response to at least three of the five questions was considered significant. Shirmer's tests before and after local pontocaine anesthesia were completed in 15 of the same patients. Less than $10 \mathrm{~mm}$ of filter paper wetting before local anesthesia was considered to reflect a significant decrease in the rate of reflex tear production. Less than $5 \mathrm{~mm}$ after local anesthesia was considered to reflect a significant decrease in the basal tear secretion rate. Slit lamp and fluorescein staining examinations were also completed in these 15 patients. Nine patients agreed to have punch biopsies taken from the mucosa of the lower lip which were evaluated histologically by standard methods.

\section{RESULTS}

Clinical features. The mean age of the entire group was $41 \pm 13 \mathrm{yr}$, with a range from 21 to $73 \mathrm{yr}$. 21 were female and 5 were male. All patients were Caucasoid. The age and sex distribution of the annular and the papulosquamous subgroups were not significantly different. 14 patients $(54 \%)$ had four or more of the preliminary American Rheumatism Association (ARA) criteria for classification as systemic LE (seven annular and seven papulosquamous). If the three cutaneous ARA criteria (facial erythema, discoid lesions, and photosensitivity) were excluded, then only five patients had four or more ARA criteria (one annular and four papulosquamous). SCLE has been present in $\sim 10 \%$ of the LE patients seen by us over the past $10 \mathrm{yr}$.
HLA typing. As shown in Table I, Al was the only HLA-A antigen that was significantly increased in frequency, occurring in 8 of the 11 annular SCLE patients $(P c<0.05)$. HLA-B8 was significantly increased in frequency in the annular $(81 \%, P c<0.007)$ and combined patient groups $(65 \%, P c<0.004)$. Although HLA-B8 was increased in frequency in the papulo-

TABLE I

HLA in SCLE

\begin{tabular}{|c|c|c|c|c|}
\hline \multirow[b]{3}{*}{ HLA } & \multicolumn{4}{|c|}{ Antigen frequency } \\
\hline & \multirow[b]{2}{*}{$\begin{array}{c}\text { Caucasian } \\
\text { controls } \\
(n>100)\end{array}$} & \multicolumn{3}{|c|}{ SCLE } \\
\hline & & $\begin{array}{l}\text { Annular } \\
(n=11)\end{array}$ & $\begin{array}{l}\text { Papulo- } \\
\text { squamous } \\
(n=15)\end{array}$ & $\begin{array}{c}\text { Combined } \\
(n=26)\end{array}$ \\
\hline & \multicolumn{4}{|c|}{$\%$ positive } \\
\hline Al & 33 & $73^{*}$ & 27 & 46 \\
\hline A2 & 51 & 27 & 40 & 35 \\
\hline A3 & 34 & 18 & 47 & 35 \\
\hline Al1 & 7 & 0 & 13 & 8 \\
\hline AW24 & 14 & 18 & 33 & 27 \\
\hline A28 & 3 & 0 & 7 & 4 \\
\hline A29 & 4 & 11 & 0 & 4 \\
\hline AW30 & 6 & 11 & 7 & 8 \\
\hline AW31 & 5 & 0 & 7 & 4 \\
\hline AW32 & 5 & 0 & 7 & 4 \\
\hline B5 & 9 & 0 & 13 & 8 \\
\hline B7 & 30 & 27 & 53 & 42 \\
\hline B8 & 23 & $81 \ddagger$ & $53 \$$ & $65^{\prime \prime}$ \\
\hline B 12 & 28 & 22 & 20 & 21 \\
\hline B14 & 6 & 0 & 7 & 4 \\
\hline B 15 & 13 & 11 & 13 & 12 \\
\hline B 17 & 9 & 0 & 7 & 4 \\
\hline B 18 & 5 & 11 & 0 & 4 \\
\hline B27 & 9 & 0 & 7 & 4 \\
\hline BW35 & 13 & 9 & 13 & 12 \\
\hline B40 & 8 & 11 & 0 & 4 \\
\hline B44 & 16 & 22 & 0 & 8 \\
\hline B51 & 7 & 0 & 7 & 4 \\
\hline DR 1 & 23 & 0 & 13 & 8 \\
\hline DR2 & 31 & 27 & 53 & 42 \\
\hline DR3 & 22 & 1009 & $60^{* *}$ & $77 \ddagger t$ \\
\hline DR4 & 37 & 33 & 13 & 21 \\
\hline DR5 & 8 & 11 & 0 & 4 \\
\hline DRW6 & 10 & 11 & 13 & 12 \\
\hline DR7 & 20 & 22 & 20 & 21 \\
\hline DRW8 & 7 & 0 & 7 & 4 \\
\hline HLA-A1,B8,DR3 & 16 & $73 \$ \S$ & 27 & $46^{111}$ \\
\hline
\end{tabular}

${ }^{*} P<0.025(P c<0.05) ; \mathrm{RR}=7.1$.

$\ddagger P<0.0002(P c<0.007) ; \mathrm{RR}=26.9$.

$\S P<0.03(P c>0.05) ; \mathrm{RR}=3.8$.

" $P<0.0001(P c<0.004) ; \mathrm{RR}=6.7$.

I $P<0.00001(P c<0.00008) ; \mathrm{RR}=67.1$.

${ }^{* *} P<0.005(P c<0.04) ; \mathrm{RR}=5.4$.

† $P<0.00001(P c<0.00008) ; \mathrm{RR}=10.8$.

$\S \S P<0.00008 ; \mathrm{RR}=15.9$.

III $P<0.004 ; \mathrm{RR}=4.5$. 
squamous group, this association was not significant after correction of the $\boldsymbol{P}$ value. Thus HLA-Al-positive individuals have a RR of 7.1 for developing the annular form of SCLE, whereas those who are HLA-B8 positive have a RR of 26.9 for developing this variety of SCLE. HLA-DR3 was found to be significantly increased in frequency in all three patient groups. All of the 11 patients with annular lesions were HLA-DR3 positive $(P c<0.00008) .9$ of the patients with papulosquamous lesions $\left(60 \%, P_{c}<0.04\right)$ and 20 of the entire group $(77 \%, P c<0.00008)$ were DR3 positive. Thus, HLA-DR3-positive individuals have a high RR (10.8) for developing SCLE of either type and an even greater RR (67.1) for the annular variety. None of the SCLE patients were homozygous for HLA-DR3. The HLAA1, B8, DR3 combination, known to be associated through linkage disequilibrium, was also significantly more common in the annular $(73 \%, P<0.00008)$ and combined patient groups $(46 \%, P<0.004)$. The HLADR3 frequency was still significantly increased $\left(\chi^{2}\right.$ $=15.8$ ) in those SCLE patients who were HLA-B8 negative when compared to the HLA-B8-negative controls. The converse was not true however, HLA-B8 was not significantly increased $\left(\chi^{2}=0.2\right)$ in the HLADR3-negative SCLE patients when compared to the HLA-DR3-negative controls. Thus the increased frequency of HLA-B8 in our SCLE patients resulted from its known linkage to HLA-DR3.

Evaluation for Sjögren's syndrome. Since Sjögren's syndrome (SS) has previously been shown to be associated with HLA-B8 (18) and HLA-DR3 (19) and since SS may be present in systemic LE (SLE) patients, we felt it necessary to determine whether our SCLE patients had any evidence of SS. None of our patients initially complained of symptoms that would suggest the presence of SS. A history suggestive of dry eyes was found in only 4 of the 17 patients (24\%) who completed the questionnaire. Only two patients (12\%) gave a history suggestive of dry mouth. Only 1 of the 15 patients tested had an abnormal reflex tear secretion rate as determined by a Shirmer's test before topical anesthesia. Only 3 of the 15 had decreased basal tear secretion (Shirmer's test after anesthesia). None of the 15 patients had corneal abnormalities by slit lamp exam and fluorescein staining. Only two of the nine patients biopsied (22\%) had abnormal minor salivary gland tissue. These abnormalities consisted of sparse interstitial round cell infiltrates. In neither of these patients did the infiltrating lymphoid or plasma cells from discrete cellular aggregates. These two biopsies would be classified as grade 1 on the 0-4 grading scale described by Greenspan et al. (20). There was concurrence of either historical or objective evidence of xerophthalmia and xerostomia in only two patients, one annular and one papulosquamous. Thus the association of HLA-B8 and -DR3 with SCLE cannot be accounted for by the frequent occurrence of subclinical SS in these patients.

\section{DISCUSSION}

A number of workers have demonstrated weak but statistically significant HLA associations in different populations of SLE patients. HLA-Al was found to be present in $14 \%$ of black SLE patients in one study (7). HLA-B5 was seen in 23\% of black American SLE patients in another study (8). HLA-B8 has been found in $33 \%(9), 48 \%$ (10), and $59 \%$ (11) of different SLE populations. HLA-B15 has been seen in $46 \%$ (12) and $40 \%$ (9) of SLE patient groups. HLA-DR2 has been found in $57 \%$ (13) and 50\% (14) of SLE patient groups and finally, HLA-DR3 has been seen $46 \%$ (13), $54 \%$ (14), and 39\% (15) of different SLE populations. The HLA-B8 $(65 \%)$ and -DR3 $(77 \%)$ frequencies that we have found in our SCLE patients are higher than have been previously reported in these other LE populations. Indeed, the presence of HLA-DR3 in all 11 of our annular SCLE patients is among the strongest HLAdisease associations yet reported. Since we have excluded the possibility that these strong HLA associations were due to the concurrent presence of subclinical Sjögren's syndrome, the high frequency of HLA-B8 and -DR3 in SCLE most likely results from the fact that we have studied a homogeneous subset of LE patients. It is impossible to determine what proportion of SCLE patients were present in the SLE populations that have been previously studied. Most authors have been unable to demonstrate HLA associations with individual clinical or laboratory features of SLE. It is possible that the presence of patients with SCLE or SS in the earlier study groups could account for the weaker HLA-B8 and -DR3 associations that have been noted.

We have not found significant HLA associations in another form of cutaneous LE, chronic scarring discoid LE (21). Millard et al. (22) have found an increased incidence of HLA-B7 and HLA-B8 in discoid LE patients. In addition, they have reported that female patients with discoid LE who are HLA-B8 positive are more likely to develop SLE than HLAB8-negative female DLE patients. It is not clear from their report whether or not they classify SCLE as a form of discoid LE or as a feature of SLE. We have seen several patients who initially presented with typical localized scarring discoid LE lesions and later developed SCLE with systemic LE activity.

Several groups have found that SLE patients who have the HLA-A1, B8 phenotype are more likely to have severe systemic disease activity, particularly nephropathy $(7,23)$. Our findings do not support this concept. SCLE tends to occur in patients who generally have a more benign form of LE. The in- 
cidence of severe central nervous system or renal involvement is lower in this subset compared to that of unselected SLE populations (16). The HLA-Al, B8, DR3 phenotype was present in 8 of the 11 annular SCLE patients (73\%) and only 4 of the 15 papulosquamous patients $(27 \%)$. The annular SCLE patients tend to have even milder types of systemic illness than the papulosquamous patients.

That subset of LE patients who have SCLE can now be added to the growing list of organ specific autoimmune conditions that are associated with the HLAB8,DR3 phenotype. These conditions include SS (18, $19,24)$, Graves' disease (25), juvenile onset diabetes mellitus (26), Addison's disease (26), Celiac disease (27), dermatitis herpetiformis (28), chronic active hepatitis of the juvenile type (29), idiopathic membranous nephropathy (30), and myasthenia gravis (31). One of the unifying features in all of these conditions could be a state of heightened immune responsiveness to either self or foreign antigenic stimulation. Thus, the tissue injury that occurs at the dermalepidermal junction in SCLE might result from a genetically programmed state of cellular immune hyperresponsiveness to antigens normally elaborated in and released from the epidermis. For example, native DNA or ultraviolet light altered-DNA could be such antigens. It is also possible that the relevant epidermal antigen could be a foreign one such as a yet unidentified virus. We are currently investigating these possibilities.

\section{ACKNOWLEDGMENTS}

We would like to thank Mr. Richard O'Hara for his expert technical assistance in this project as well as Ms. Marquite Brown and Mrs. Carol Buswell for their help in preparing the manuscript.

This work was supported by grants AM-19101, AM-00730 and AI-12563 from the National Institutes of Health and a grant from the Dermatology Foundation.

\section{REFERENCES}

1. Block, S. R., J. B. Winfield, M. D. Lockshin, W. A. D'Angelo, and C. L. Christian. 1975. Studies of twins with systemic lupus erythematosus. A review of the literature and presentation of 12 additional sets. Am. J. Med. 59: 533-552.

2. Arnett, F. C., and L. E. Shulman. 1976. Studies in familial systemic lupus erythematosus. Medicine. 55: 313-322.

3. Miller, K. B., and R. S. Schwartz. 1979. Familial abnormalities of suppressor cell function in systemic lupus erythematosus. N. Engl. J. Med. 301: 803-809.

4. Kantor, F. S., A. Ojeda, and B. Benacerraf. 1963. Studies on artificial antigens. I. Antigenicity of DNP-polylysine and DNP-copolymers of lysine and glutamic acid in guinea pigs. J. Exp. Med. 117: 55-69.

5. Rose, N. R., L. D. Bacon, R. S. Sundick, Y. M. Kong, P. Esquivel, and P. E. Bigazzi. 1977. Genetic regulation in autoimmune thyroiditis. In Autoimmunity: Genetic, Immunologic, Viroligic, and Clinical Aspects. N. Talal, editor. Academic Press, Inc., New York. 3: 63-87.

6. Talal, N. 1977. Autoimmunity: Genetic, Immunologic, Virologic and Clinical Aspects. Academic Press, Inc., New York. XVII.

7. Goldberg, M. C., F. C. Arnett, W. B. Bias, and L. E. Shulman. 1976. Histocompatibility antigens in systemic lupus erythematosus. Arth. Rheumat. 19: 129-132.

8. Nies, K. M., J. C. Brown, E. L. DuBois, F. C. Quismorio, G. F. Friou, and P. I. Terasaki. 1974. Histocompatibility antigens (HL-A) and lymphocytotoxic antibodies in systemic lupus erythematosus (SLE). Arthritis. Rheum. 17: 397-402.

9. Grumet, F. C., A. Coukell, J. G. Bodmer, W. F. Bodmer, and H. O. McDevitt. 1971. Histocompatibility (HL-A) antigens associated with systemic lupus erythematosus. N. Engl. J. Med. 285: 193-196.

10. Scherak, O., G. Kolarz, and W. R. Mayr. 1978. HLA-B8 in Caucasian patients with systemic lupus erythematosus. Scand. J. Rheumatol. 7: 3-6.

11. Kampf, D., R. Malchus, M. Alexander, and I. Hoppe. 1979. HLA-antigens in systemic lupus erythematosus (SLE). Arch. Dermatol. Res. 264: 345-350.

12. Waters, H., P. Konrad, and R. L. Walford. 1971. The distribution of HL-A histocompatibility factors and genes in patients with systemic lupus erythematosus. Tissue Antigens. 1: 68-73.

13. Reinertsen, J. L., J. H. Klippel, A. H. Johnson, A. D. Steinberg, J. L. Decker, and D. L. Mann. 1978. Blymphocyte alloantigens associated with systemic lupus erythematosus. N. Eng. J. Med. 99: 515-518.

14. Gibofsky, A., R. J. Winchester, M. Patarroyo, M. Fotino, and H. G. Kunkel. 1978. Disease associations of the Ialike human alloantigens. Contrasting patterns in rheumatoid arthritis and systemic lupus erythematosus. J. Exp. Med. 148: 1728-1732.

15. Scherak, O., J. S. Smolen, and W. R. Mayer. 1979. Prevalence of HLA-DRW2 not increased in systemic lupus erythematosus. N. Engl. J. Med. 301: 612 .

16. Sontheimer, R. D., J. R. Thomas, and J. N. Gilliam. 1979. Subacute cutaneous lupus erythematosus. A cutaneous marker for a distinct lupus erythematosus subset. Arch. Dermatol. 115: 1409-1415.

17. Feighery, C. F., and P. Stastny. 1980. Cell mediated cytotoxicity against HLA-D-region products expressed in monocytes and B lymphocytes. Immunogenetics. 10: $31-38$.

18. Fye, K. H., P. I. Terasaki, H. M. Moutsopoulous, T. E. Daniels, J. P. Michalski, and N. Talal. 1976. Association of Sjögren's syndrome with HLA-B8. Arthritis Rheum. 19: 883-886.

19. Chused, T. M., S. S. Kassan, G. Opelz, H. M. Moutsopoulous, and P. I. Tarasaki. 1977. Sjögren's syndrome associated with HLA-DW3. N. Engl. J. Med. 296: 895-897.

20. Greenspan, J. S., T. E. Daniels, N. Talal, and R. A. Sylvester. 1974. The histopathology of Sjögren's syndrome in labial salivary gland biopsies. Oral Pathol. 37: 217-229.

21. Prystowsky, S. D., and J. N. Gilliam. 1975. Discoid lupus erythematosus as a part of a larger disease spectrum. Arch. Dermatol. 111: 1448-1452.

22. Millard, L. G., N. R. Rowell, and S. M. Rajah. 1977. Histocompatibility antigens in discoid and systemic lupus erythematosus. Br. J. Dermatol. 96: 139-144. 
23. Rigby, R. J., R. L. Dawkins, J. D. Wetherall, and B. R. Hawkins. 1978. HLA in systemic lupus erythematosus: influence on severity. Tissue Antigens. 12: 25-31.

24. Ivanyi, D., E. Hincova, K. Sula, I. Drizhal, E. Erbenova, H. Macurova, C. Dostal, J. Horejs, J. Balik, and J. Juran. 1976. Increased frequency of HLA-B8 and HLA-DRW3 in Sjögren's syndrome. In HLA and Disease. J. Dausset and A. Svejgatard, editors. Inserm, Paris. 202.

25. McMichael, A. J., T. Sasazuki, F. C. Grumet, R. Payne, and H. O. McDevitt. 1976. An HL-A8 associated LD antigen in patients with Graves disease. In: Histocompatibility Testing 1975. F. Kissmeyer-Nielsen, editor. Munksgaard, Copenhagen. 769-772.

26. Thomsen, M., P. Platz, O. Anderson, M. Christy, J. Lyngsoe, J. Nerup, K. Rasmussen, L. P. Ryder, L. StaubNielsen, and A. Svegaard. 1975. MLC typing in juvenile diabetes mellitus and idiopathic Adison's disease. Transplant. Rev. 22: 125-147.
27. Keuning, J. J., A. S. Pena, J. P. van Hooff, A. van Leeuwen, and J. J. van Rood. 1976. HLA-DW3 associated with coeliac disease. Lancet. I: 506-508.

28. Katz, S. I., Z. M. Falchuk, M. V. Dahl, G. N. Rogentine, and W. Strober. 1972. HL-A8: a genetic link between dermatitis herpetiformis and gluten sensitive enteropathy. J. Clin. Invest. 51: 2977-2980.

29. Ryder, L. P., and A. Svejgaard. 1976. Association between HLA and disease. Report from the HLA and Disease Registry of Copenhagen. Published by the authors in Copenhagen.

30. Klouda, P. T., E. J. Acheson, F. S. Golby, W. Lawler, J. Manos, P. A. Dyer, R. Harris, and N. P. Mallick. 1979. Strong association between idiopathic membranous nephropathy and HLA-DRW3. Lancet. II: 770-771.

31. Kaakinen, A., R. Pirskanen, and A. Tiilikainen. 1975. LD antigens associated with HL-A8 and myasthena gravis. Tissue Antigens. 6: 175-182. 